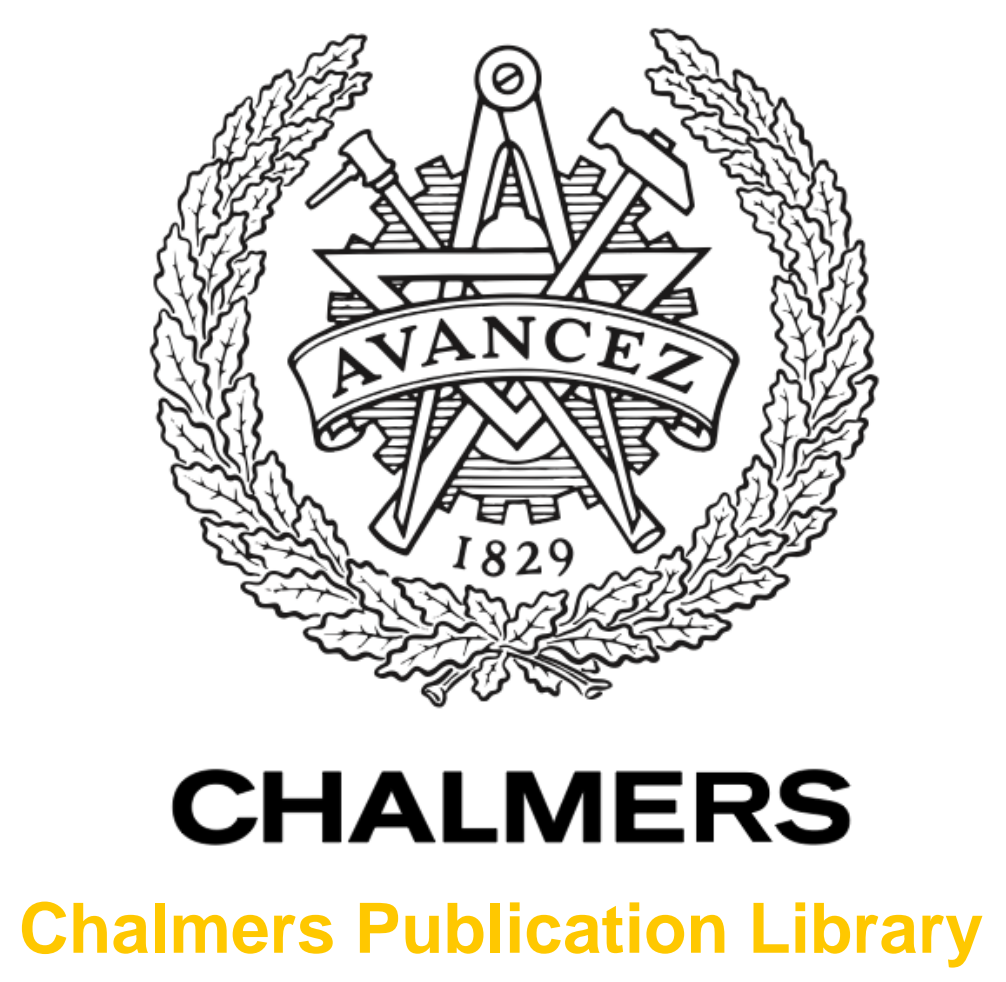

\title{
Design of a simple transition from microstrip to ridge gap waveguide suited for MMIC and antenna integration
}

This document has been downloaded from Chalmers Publication Library (CPL). It is the author's version of a work that was accepted for publication in:

IEEE Antennas and Wireless Propagation Letters (ISSN: 1536-1225)

Citation for the published paper:

Zaman, A. ; Vukusic, T. ; Alexanderson, M. (2013) "Design of a simple transition from microstrip to ridge gap waveguide suited for MMIC and antenna integration". IEEE

Antennas and Wireless Propagation Letters, vol. 12 pp. 1558 â 1561.

http://dx.doi.org/10.1109/LAWP.2013.2293151

Downloaded from: http://publications.lib.chalmers.se/publication/193104

Notice: Changes introduced as a result of publishing processes such as copy-editing and formatting may not be reflected in this document. For a definitive version of this work, please refer to the published source. Please note that access to the published version might require a subscription.

Chalmers Publication Library (CPL) offers the possibility of retrieving research publications produced at Chalmers University of Technology. It covers all types of publications: articles, dissertations, licentiate theses, masters theses, conference papers, reports etc. Since 2006 it is the official tool for Chalmers official publication statistics. To ensure that Chalmers research results are disseminated as widely as possible, an Open Access Policy has been adopted.

The CPL service is administrated and maintained by Chalmers Library. 


\title{
Design of a Simple Transition from Microstrip to Ridge Gap Waveguide Suited for MMIC and Antenna Integration
}

\author{
Ashraf Uz Zaman, Tin Vukusic, Mats Alexanderson, Member, IEEE, Per-Simon Kildal, Fellow,IEEE
}

\begin{abstract}
This paper describes a simple and low-loss microstrip-to-ridge gap waveguide transition with a very compact geometry. The transition transforms the EM fields from the microstrip mode to the air-filled ridge gap waveguide mode. This is achievable if the height of the air gap in the ridge gap waveguide is kept almost equal to the thickness of the substrate of the microstrip line. The transition has a pressure contact between the ridge and the microstrip line, so it works without soldering. This is advantageous in systems which require mechanically separable split-blocks or modules and need a lot of transitions. Experimental results of the manufactured back-toback transition show an insertion loss of $0.32 \mathrm{~dB}$ and a return loss of $\mathbf{1 4 . 1 5} \mathrm{dB}$ over $55 \%$ relative bandwidth in Ka band.
\end{abstract}

Index Terms-Microstrip transition, ridge gap waveguide, MMIC integration, antenna array.

\section{INTRODUCTION}

$\mathrm{L}$ OW-LOSS planar antenna technology has gained a lot of interest very recently due to upcoming commercial applications at high frequency and millimeter-wave frequency ranges. Conventional waveguide slot array antennas are attractive candidates for high frequency applications requiring high gain [1-3]. However, waveguide slot arrays have typically very complex feed networks under the radiating slots, and manufacturing costs are large at high frequency because there must be good electrical contact between the slotted plate and the feed structure [4-5]. On the other hand, planar microstrip antenna solutions are cost effective, but unfortunately their distribution networks are lossy and suffer from unwanted leakage through surface-waves [6-7]. To address these issues, the gap waveguide technology introduced in [8-9] can be applied very successfully. Gapwaveguide structures do not have critical electric contact

Manuscript received 19 February, 2013. This work has been supported in part by the Swedish Research Council VR, and by The Swedish Governmental Agency for Innovation Systems (VINNOVA) within the VINN Excellence Center Chase.

A.Uz Zaman and P.-S. Kildal are with Department of Signals and Systems, Chalmers University of Technology, SE-412 96 Göteborg, Sweden.(e-mail: zaman@ chalmers.se; per-simon.kildal@chalmers.se)

T. Vukusic and M. Alexanderson and are with R\&D section of Trebax AB, SE-421 31 Västra Frölunda, Göteborg, Sweden (e-mail: tin@trebax.se; mats@trebax.se). requirement and suppress all surface waves or parallel plate modes. Unlike the waffle-iron structures described in [10], this technology uses the basic cut-off of a PEC-PMC parallelplate waveguide configuration to control and guide desired electromagnetic wave propagation between the two parallel plates. In the gap waveguide, the PMC condition must be emulated by an artificial magnetic conductor (AMC) in the form of a periodic structure such as metal pins or mushroom [11-12]. The AMC layer and the top metal layer together create a stopband for parallel-plate (PP) modes. All global parallel-plate (PP) modes are in cutoff within this stopband. However, if the AMC layer incorporates a guiding structure such as ridge, groove or strip, EM waves can propagate along them without being leaked from the structure. In that case, such parallel-plate structures can be used as effective transmission lines [13]. Using the gap waveguide concept and metal pin surface as AMC, microwave components such as high $\mathrm{Q}$ bandpass filters and rat race hybrids have been designed [14-15]. Low loss gap waveguide technology can also have advantages for antenna applications. In particular, the distribution networks can be made with lower loss because gap waveguides do not require dielectric substrates. Apart from that, it is possible to increase the line dimensions (compared to microstrip case) considerably in a way that no surface waves will propagate and even the conductive losses will become smaller. Also, antennas can be made without any metal contact between the top metal plate with radiating slots and the bottom AMC plate containing the topology of the distribution network. Moreover, such split-block gap waveguide structures are quite suitable for assembly and packaging of millimeter-wave circuits. With these advantages in mind, some antennas based on gap waveguide technology have been designed and presented [16-18].

Successful integration of antenna and MMIC based active $\mathrm{RF}$ circuit is also critical in designing microwave modules at millimetre wave frequencies. Therefore, good interfaces between different components are needed. Many high frequency TX/RX modules contain MMIC chips, and they have typically microstrip line both at input and output. Therefore, a good transition between microstrip line and ridge gap waveguide is a key element for good integration. Such transitions should be compact and should operate over as large bandwidth as possible. This can only be achieved by accurate impedance matching and mode transformation between these two different transmission lines. The purpose of this work is 
to present a simple and compact transition between microstrip line and ridge gap waveguide. In the proposed design, the microstrip line is placed in line with the ridge section of the gap waveguide, thereby allowing a gradual mode transformation from the typical microstrip mode to the airfilled ridge gap waveguide mode. The microstrip substrate height should be equal to the air gap size, but this usually falls within the range of normal microstrip substrate thicknesses in the appropriate frequency bands. Thus, the substrates can be selected with the thickness available from the manufacturer and it can have any dimension in lateral directions.

\section{TRANSITION CONCEPT}

A microstrip to ridge gap waveguide transition has been investigated recently in [19]. The concept was based on electromagnetic coupling where a microstrip patch had been used. The present transition operates over larger relative bandwidth and shows lower loss than the transition presented in [19]. It is based on the fact that the E- field of the dominant mode in a microstrip line can be easily transformed to a standard ridge waveguide mode [20]. It is even simpler for ridge gap waveguides, because their dominant mode is a quasi-TEM mode similar to that of a microstrip line. This is shown in fig. 1 .
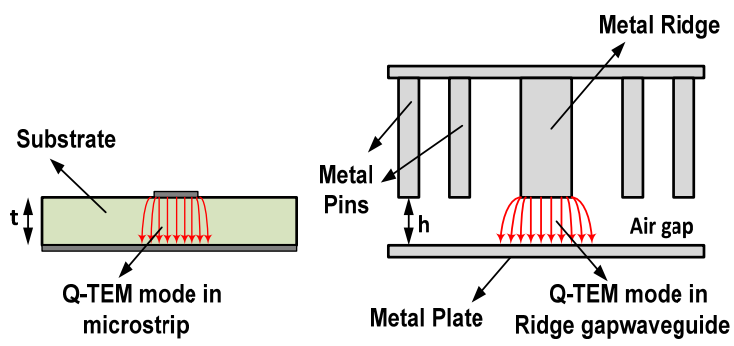

Fig. 1. E-field distributions of the dominant mode of microstrip line and ridge gap waveguide.

Due to the similar field distribution, the only requirement for a good transition between these two different lines is essentially an interface for transforming E-fields in the dielectric to E-fields in the air. This can be done by tapering down the width of the ridge section (that is wider $50 \Omega$ line without dielectric) in steps to the same width as the microstrip line. In the proposed design, this is done with one step, and this extension of the ridge is placed above the end of the microstrip line, covering it. The extended tapered ridge section needs to be in electrical contact with the microstrip line on the substrate. This can be achieved by soldering, gluing or simply by pressing the ridge section down. In our work, the electric contact is achieved only by mechanical pressure contact. No soldering is used for achieving the metal contact between these two sections of the transition. This is desirable in many applications as it opens up opportunity for replacing or changing the faulty MMICs. Also, soldering of such transitions may sometime cause poor repeatability, longer assembly time and higher cost [21]. A back-to-back configuration of the designed transition between the microstrip line and the ridge gap waveguide is shown in fig. 2 . The single tapered section of the ridge is used for the gradual transition of the E-field from the microstrip mode in substrate to the quasi-TEM mode of the air-filled ridge gap waveguide. A standard Chebychev transformer based on several $\lambda_{\mathrm{g}} / 4$ sections of different widths can also be employed to improve the transition. However, the main focus in this work is not only to design a good quality transition but also to design the transition in a compact form to make it suitable for MMIC integration. That is the main motivation for using a single step transition of the ridge. Usually, any kind of discontinuity or transition tends to radiate at high frequency. To tackle this problem, two pins with shorter heights are placed after the tapered ridge section just above the end of the microstrip line. These two pins will act as PMC packaging technique as described in [22] and will suppress any kind of radiation leakage around the discontinuity.

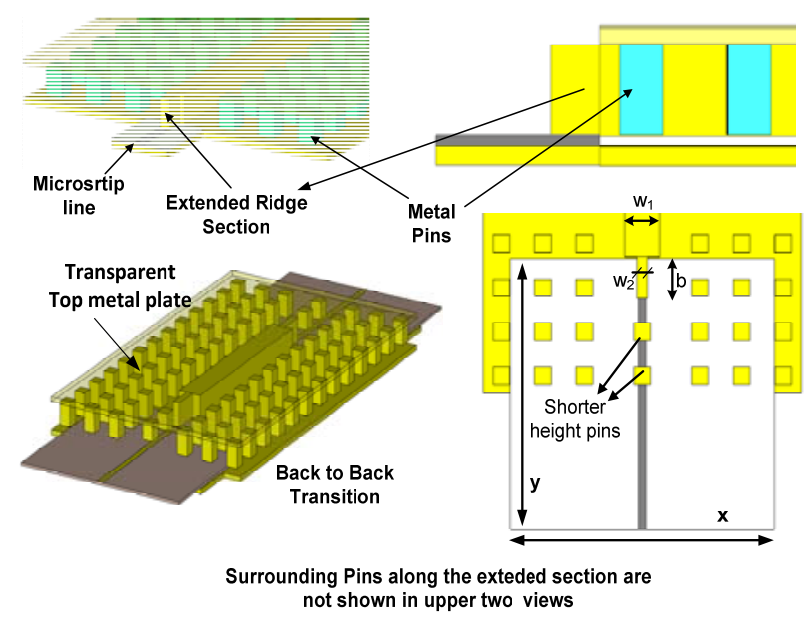

Fig. 2. Drawing of two pieces of the the proposed transition $\mathrm{W}_{1}=2.65 \mathrm{~mm}$, $\mathrm{W}_{2}=0.72 \mathrm{~mm}, \mathrm{x}=\mathrm{y}=20 \mathrm{~mm}$ and $\mathrm{b}=2.75 \mathrm{~mm}$. Note that the top metal plate to which the pins are fastened is made transparent to reveal the details of the transition.

\section{Simulation Results}

The ridge gap waveguide section used in this work is designed to operate in $\mathrm{Ka}$ band, from 26 to $40 \mathrm{GHz}$. We have used periodic metal pins to design the required AMC surface. The dimensions of the periodic metal pin structure are chosen according to the guidelines given in [23]. For the pin dimensions given in Fig.3, the stopband ranges from 22 to $46 \mathrm{GHz}$. Here, the dispersion diagram is computed for a unit cell of the periodic pin surface, without any dielectric material present. Still, in a real situation, there will be a loading effect due to the presence of the substrate of the microstrip line. This loading effect will change the upper cutoff frequency of the parallel plate modes and will reduce the stop-band. However, this reduction will not be significant because the stop-band is already quite large.

Two transitions are simulated together in a back-to-back configuration. The two substrates used for the input and output microstrip lines are Arlon CLTE-AT $\left(\varepsilon_{\mathrm{r}}=3.0\right)$ with thickness $t=0.254 \mathrm{~mm}$, and size of $20 \times 20 \mathrm{~mm}^{2}$. These two substrates are glued on a common metal plate. The size of the substrate is chosen a bit bigger than needed to have a stable setup for measuring the back- to-back transition. The width of the $50 \Omega$ microstrip line has been chosen as $0.65 \mathrm{~mm}$. The air 
gap height ' $h$ ' for the ridge gap waveguide section should be the same as substrate thickness ' $t$ ' for the microstrip. However, the thickness of the metal layer on the substrate and the added thickness due to glue are taken into account in the simulation, and therefore the value of ' $h$ ' is chosen to be 0.43 $\mathrm{mm}$. The chosen length of the ridge gap waveguide section is about $42 \mathrm{~mm}$, which is approximately four wavelengths at $30 \mathrm{GHz}$.

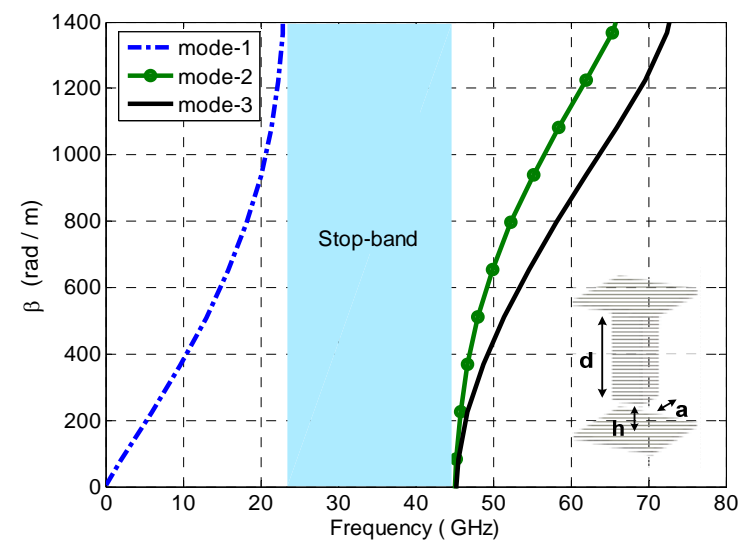

Fig. 3. Dispersion diagram for unit-cell, $\mathrm{a}=0.8 \mathrm{~mm}, \mathrm{~h}=0.43 \mathrm{~mm}, \mathrm{~d}=2.35$ $\mathrm{mm}$.

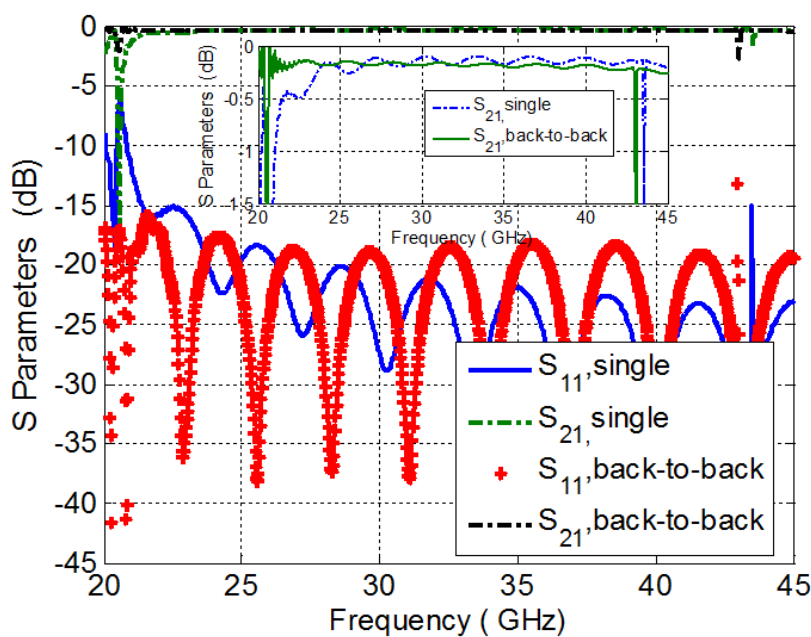

Fig. 4. Simulated performance of proposed single transition as well as backto-back transition.

CST microwave time-domain solver was used for the simulations. The result for both the single transition and the back-to-back transition is shown in fig. 4 . The periodic peaks in the $S_{11}$ of the back-to-back transition are caused by the interference between the two transitions at both sides. Resonance peaks below $23 \mathrm{GHz}$ and after $43 \mathrm{GHz}$ are attributed to parallel- plate modes outside the designed stopband. As mentioned before, the upper frequency limit of the stop-band has been shifted from $46 \mathrm{GHz}$ to around $43 \mathrm{GHz}$ due to the loading effect of the dielectric substrate.

\section{EXPERIMENTAL RESULTS}

In order to validate the concept of the transition, the simulated back-to-back transition with only one tapered section of the ridge was manufactured and measured. The machined ridge gap waveguide section with the pin structure was fabricated separately using a computer numerically controlled $(\mathrm{CNC})$ milling machine. The other metal part is a smooth metal plate on which the two PCBs with the microstrip input and output ports are glued by epoxy resin. These two building blocks are held together with some screws and are mechanically pressed on top of each other by tightening the screws. The two building blocks can be separated from each other for maintenance or other purposes, and reassembled again.

During measurements, the back-to-back transition was connected to Anritsu 36397C Vector Network Analyzer (VNA) via a universal test fixture (UTF) from Rosenberger for launching the signal for S-parameter measurements. Standard TRL calibration was done to remove the effects of the connectors and to move the measurement plane from the $2.4 \mathrm{~mm}$ connectors to the microstrip plane. This also calibrates out the losses of the microstrip line sections at the input and output side. However, the conductor loss in the ridge gap waveguide section has not been calibrated out and this loss is visible in the measured responses. The measured responses for the back-to-back transition are shown in fig.5 and the pictures of the manufactured prototype are shown in fig.6.

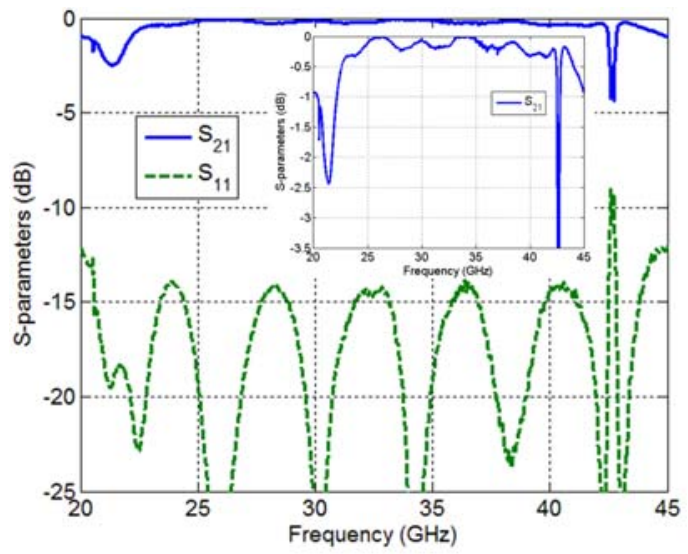

Fig. 5. Measured results for the proposed back-to-back transition, line length is approximately $2 \lambda$ at $30 \mathrm{GHz}$

As shown in fig. 5 , the measured $\mathrm{S}_{11}$ is $-14.15 \mathrm{~dB}$ over 23 $43 \mathrm{GHz}$ band, which means that a single transition will have even lower value of $S_{11}$ over the entire Ka band of interest. The maximum insertion loss over the same $23-43 \mathrm{GHz}$ band is found to be $0.32 \mathrm{~dB}$ for the back-to-back transition including the losses of the ridge gap waveguide section. This means a loss smaller than $0.16 \mathrm{~dB}$ per single transition over the entire $\mathrm{Ka}$ band. The measured $\mathrm{S}_{11}$ for the back-to-back transition is about $4.1 \mathrm{~dB}$ larger than the simulated value. The measured $\mathrm{S}_{21}$ is about $0.13 \mathrm{~dB}$ lower than the simulated $\mathrm{S}_{21}$. These differences originate from tolerances. In simulations, a perfect alignment and positioning between the two parts of the transition was used, whereas in practice, there is always tolerances and human error involved during measurements. Also, all kind of losses such as tan $\delta$, surface imperfections etc. cannot be accurately accounted for in full-wave simulations. However, the return loss and insertion loss remain within 
acceptable limits and are in reasonable agreement with the simulated results presented in fig.4. For testing repeatability, different parts of the transition are dismounted and remounted several times, and each time the measured results were found very similar to that presented in fig.5.

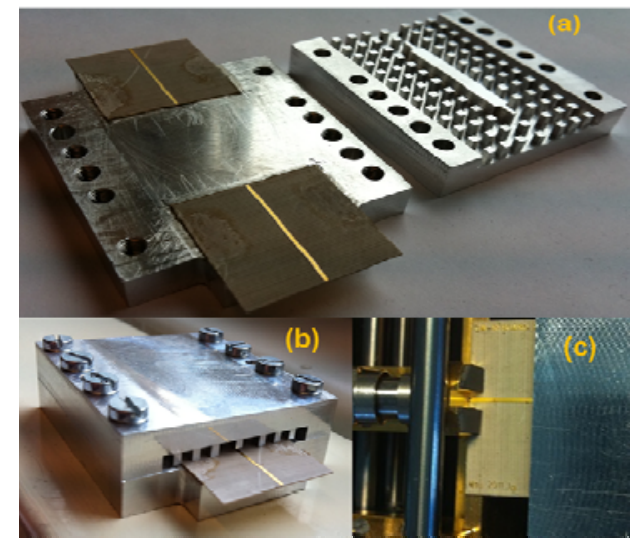

Fig. 6. Prototype of proposed back-to-back transition: a) two separate sections of the transition; (b) Two sections screwed together and (c) launching probes of the UTF.

\section{CONCLUSION}

A simple transition from microstrip to ridge gap waveguide has been designed for integration of MMIC based RF circuits with gap waveguide-fed antennas. The microstrip mode is effectively transformed into the air-filled ridge gap waveguide mode by means of a narrow extended section of the ridge placed on top of the microstrip line. The height of the air-gap of the ridge gap waveguide section is kept close to the thickness of microstrip substrate. S-parameter measurements of the back-to-back transition are in good agreement with the simulated results. An additional feature of the proposed transition is that- there is no need for soldering or gluing. This can be of advantage in many applications where it is desirable to dismount or replace components. Though designed for MMIC and ridge gap waveguide antenna integration, this simple transition can be used in other cases such as for integrating diplexer filter with MMIC.

\section{ACKNOWLEDGMENT}

The authors would like to thank Tomas Östling and Johan Carlsson of Arkivator AB, Göteborg, Sweden for their help in manufacturing the prototype used in measurements.

\section{REFERENCES}

[1] E.Levine, G. Malamud, S.Shtrikman and D. Treves, " A study of microstrip array antennas with the feed network", IEEE Transection on Antennas and Propagation, vol.37, no.4, pp.426-464, April 1989.

[2] J. Hirokawa and M. Ando, "Single-layer feed waveguide consisting of posts for plane TEM wave excitation in parallel plates", IEEE Trans. Antennas Propag., vol. 46, no. 5, pp. 625-630, May 1998.

[3] J. Hirokawa and M. Ando, "Efficiency of $76 \mathrm{GHz}$ Post-wall Waveguidefed Parallel-plate Slot Arrays", IEEE Trans. Antennas Propag., vol. 48 , no. 11, pp. 1742-1745, November 2000.

[4] YKimura, T.Hirano, J.Hirokawa and M.Ando, "Alternating-phase fed single-layer slotted waveguide arrays with chokes dispensing with narrow wall contacts", IEE Proc.-Microw. Antennas Propag, Vol. 148, No 5, October 2001.
[5] D. Liu, B.Gaucher, U. Pfeiffer and J.Grzyb, "Advanced Millimeter-wave Technologies-Antennas, Packaging and Circuits", Chapter-6, John Wiley \& Sons Publication, ISBN 9780470996171.

[6] D M. Pozar, “ Considerations for Millimeter Wave Printed Antennas", IEEE Transection on Antennas and Propagation, vol.31, no.5, pp.740747, September 1983.

[7] E.Levine, G. Malamud, S.Shtrikman and D. Treves, "A study of microstrip array antennas with the feed network", IEEE Transection on Antennas and Propagation, vol.37, no.4, pp.426-464, April 1989.

[8] P.-S. Kildal, A. Uz Zaman, E. Rajo-Iglesias, E. Alfonso, A. ValeroNogueira, "Design and experimental verification of ridge gap waveguides in bed of nails for parallel plate mode suppression", IET Microwaves, Antennas \& Propagation, Vol.5, No. 3, pp. 262-270, March 2011.

[9] A. Uz. Zaman, P.-S. Kildal, M. Ferndahl, A. Kishk,"Validation of Ridge Gap Waveguide Performance Using in-house TRL Calibration Kit", 4th European Conference on Antennas and Propagation, EuCAP 2010, Barcelona, April 2010.

[10] C.G. Matthaei, L. Young, and E. M. T. Jones, "Microwave Filters, Impedance Matching Networks, and Coupling Structures", New York, McGraw-Hill, 1964.

[11] M.G. Silveirinha, C.A.Fernandes and J.R. Costa, "Electromagnetic characterization of textured surfaces formed by metallic pins," IEEE Trans. Antennas Propagat, vol. 56, no. 2, pp. 405-415, February 2008.

[12] D.Sievenpiper, L.Zhang, R.F.J.Broas, N.G.Alexopolous, and E. Yablonovitch, "High- impedance electromagnetic surfaces with a forbidden frequency band," IEEE Trans. Microwave Theory Tech., vol. 47, no. 11, pp. 2059-2074, November 1999.

[13] P.-S. Kildal, "Three metamaterial - based gap-waveguides between parallel metal plates for mm /submm waves,' 3rd European Conference on Antennas and Propagation, Berlin, March 2009.

[14] A. Uz Zaman, P.-S. Kildal, and A.Kishk, "Narrow-Band Microwave Filter Using High Q Groove Gap Waveguide Resonators with Manufacturing Flexibility and no Sidewalls," IEEE Transactions on Components, Packaging and Manufacturing Technology, Vol.2, No-11, pp- 1882-1888, November, 2012.

[15] H. Raza and J.Yang, "A Low Loss Rat Race Balun in Gap Waveguide Technology," 5th European Conference on Antennas and Propagation (EUCAP), Rome, pp. 1230-1232, April 2011.

[16] Ahmed Kishk and P.-S. Kildal," Quasi-TEM H-plane Horns with Wideband Open Hard Side-Walls," 4th European Conference on Antennas and Propagation, 2010, Barcelona, Spain, April 12-16, 2010.

[17] H. Kirino and K. Ogawa, "A $76 \mathrm{GHz}$ Multi-Layered Phased Array Antenna Using a Non-Metal Contact Metamaterial Waveguide", IEEE Transactions on Antennas and Propagation, Vol. 60, No. 2, pp. 840853, February, 2012.

[18] A Uz Zaman and P.-S. Kildal, "Slot Antenna in Ridge Gap Waveguide Technology," 6th European Conference on Antennas and Propagation, Prague , March, 2012

[19] A. A. Brazález, A. Uz Zaman and P.-S. Kildal, "Investigation of a Microstrip-to-Ridge Gap Waveguide transition by electromagnetic coupling" IEEE Antenna and Propagation Society Symposium, pp. 1-2, July 2012.

[20] H.-W. Yao, A. Abdelmonem, J.-F. Liang, and K. A. Zaki, "Analysis and design of microstrip-to-waveguide transitions," IEEE Trans. Microwave Theory and Technique, vol. 42, no. 12, pp. 2371-2380, Dec. 1994

[21] Y. Zhang, J. A. Ruiz-Cruz, K. A. Zaki and A. J. Piloto, " A Waveguide to Microstrip Inline Transition With Very Simple Modular Assembly," IEEE Microwave and Wireless Components Letters, Vol. 20, No.9, September 2010.

[22] A. Kishk, A. Uz Zaman, and P.-S. Kildal, "Numerical Prepackaging with PMC lid - Efficient and Simple Design Procedure for Microstrip Circuits including the Packaging," ACES Applied Computational Society journa, vol. 27, no. 5, pp. 389-398, May 2012.

[23] E. Rajo-Iglesias and P.-S. Kildal, "Numerical studies of bandwidth of parallel plate cut-off realized by bed of nails, corrugations and mushroom-type EBG for use in gap wave-guides," IET Microwaves, Antennas \& Propagation, vol. 5, no. 3, pp. 282-289, March 2011. 